\title{
Flora vascular de la Sierra Monte Grande, municipio de Charcas, San luis Potosí, México
}

\author{
J. Antonio Reyes Agüero', Francisco González Medrano ${ }^{2}$ \\ y José D. García Pérez ${ }^{1}$
}

\begin{abstract}
'Área de Etnobotánica, Programa de Botánica, Instituto de Recursos Naturales, Colegio de Postgraduados, 56230 Chapingo, México. e-mail: etnobot@colpos.colpos.mx

2Departamento de Botánica, Instituto de Biología, Universidad Nacional Autónoma de México, Apdo. Postal 70-233, Cd. Universitaria, Delegación Coyoacán, 04510 México, D.F., México.
\end{abstract}

\begin{abstract}
Resumen. La Sierra Monte Grande tiene una extensión de $41.8 \mathrm{~km}^{2}$. Se localiza en la zona norte del altiplano potosino; su flora vascular está compuesta por 74 familias, 242 géneros y 397 especies que se encuentran en los siguientes tipos de vegetación: matorrales crasicaule, desértico micrófilo y desértico rosetófilo, así como encinar arbustivo y piñonar. Se consignan 45 registros inéditos para la región del altiplano potosino.

Palabra clave: encinar, matorrales xerófilos, nuevos registros, piñonar, riqueza específica.

Abstract. Monte Grande mountain range is a small $\left(41.8 \mathrm{~km}^{2}\right)$ area located in the north San Luis Potosi high plateau. The characteristic vegetation include oakscrub, pinyon woodland and three types of xerophytic scrub. The flora of this area comprises 74 families, 242 genera and 397 species; 45 of these species represent new records for the San Luis Potosi high plateau.
\end{abstract}

Key words: new records, oakscrub, pinyon, species richness, woodland, xerophitic scrub.

$\mathbf{L}$

a flora de las zonas áridas y semiáridas del norte de México ha sido objeto de diversos estudios desde el siglo pasado. Según Rzedowski (1965), J. L. Berlandier, en 1827, fue el primer botánico que exploró el altiplano potosino; después de este naturalista siguieron muchos otros, como G. Schaffner, H. Galeotti, E. Palmer, I. Palacios y G. Barroeta, en el siglo pasado, y C. Lundell, I. M. Johnston, M. C. Johnston, E. Matuda, E. Hernández X. y H. Bravo, en la primera mitad de este siglo. Para la segunda mitad destacaron, por parte de la Universidad Autónoma de San Luis Potosí, J. Rzedowski y G. Calderón, quienes entre 1954 y 1960 estudiaron la flora y la vegetación de San Luis Potosí (Calderón, 1960; Rzedowski, 1955, 1956, 1957, 1959, 1960, 1965; Rzedowski y Calderón, 1957).

Desde fines de la década de los años sesenta y hasta la mitad de la presente, personal de la Escuela Nacional de Agricultura (hoy Universidad Autónoma Chapingo) y del Colegio de Postgraduados en Ciencias Agrícolas iniciaron una serie de trabajos, de ecología aplicada. En cada una de estas investigaciones se incluyó un catálogo de especies vegetales presentes en los diferentes sitios de estudio (Aguirre, 1970;
Becerra, 1971, entre los primeros trabajos, y Molina, 1990; Robledo, 1990 ; Balleza, 1993, entre los últimos). En esa misma época también se realizaron algunos trabajos florísticos por familias (cactáceas, compuestas y leguminosas) por parte de investigadores de la Universidad Autónoma de San Luis Potosí (Hernández, 1985; Salas, 1987; Sánchez, 1989).

La zona sur de la Sierra Monte Grande fue incluida en un estudio de Rzedowski (1955) sobre las diferencias florísticas y ecológicas condicionadas por ciertos tipos de substrato geológico en San Luis Potosí. Dicho autor colectó en la zona sur de Monte Grande 85 especies distribuidas en el chaparral (encinar arbustivo) y en el zacatal. El área también ha sido visitada por botánicos de la Universidad Autónoma de San Luis Potosí, del Herbario Nacional Forestal y del Colegio de Postgraduados, de acuerdo con registros encontrados en los herbarios correspondientes.

El objetivo del presente trabajo es la elaboración de un catálogo de especies de plantas vasculares de la Sierra Monte Grande, y analizar cuántos y cuáles podrían ser nuevos registros para la flora del altiplano potosino. 


\section{Área de estudio}

Localización y delimitación. La Sierra Monte Grande, conocida también como Picacho de Lajas, tiene una extensión de $41.8 \mathrm{~km}^{2}$ y se localiza en la zona norte del altiplano potosino (figura 1), a unos $20 \mathrm{~km}$ al noroeste de la cabecera municipal de Charcas, S.L.P.; las coordenadas geográficas son: $101^{\circ} 07^{\prime} 6{ }^{\prime \prime}-101^{\circ} 11^{\prime} 16^{\prime \prime}$ de longitud oeste, y $23^{\circ} 17^{\prime} 43^{\prime \prime}-23^{\circ} 21^{\prime} 34^{\prime \prime}$ de latitud norte (Anónimo, 1971); colinda al este con la sierra Picachos del Tunalillo y ambas están separadas, aproximadamente $25 \mathrm{~km}$ hacia el noreste, de la serranía de Real de Catorce (Anónimo, 1975).

Topografia y litología superficial. La topografía de Monte Grande es accidentada. El gradiente altitudinal abarca un intervalo de 2,200 a $2,800 \mathrm{~m}$, y son frecuentes las pendientes de $30 \%$ o más; muchas laderas presentan exposiciones hacia el noroeste. En la zona oriental domina la caliza y en la zona occidental roca ígnea extrusiva; en la parte más alta de la sierra se encuentra roca ígnea extrusiva ácida, mientras que en las porciones inferiores existe material aluvial (Anónimo, 1971; Flores, 1985).

Clima. El clima para la parte baja del área estudiada corresponde al más seco de los semisecos $\left(\mathrm{BS}_{\mathrm{o}} \mathrm{k}\right)$. La temperatura media anual es de $14.8^{\circ} \mathrm{C}$; la precipitación es de $363 \mathrm{~mm}$ anuales de lluvia; la máxima precipitación se presenta entre mayo y septiembre. La evaporación es del orden de 2,181 $\mathrm{mm}$ anuales, con la máxima entre marzo y julio. El período de heladas se presenta, por lo general, desde los últimos días de septiembre hasta fines de marzo. El clima de la parte alta de la sierra es más húmedo, con presencia frecuente de neblinas, pero se carece de registros climatológicos (Anónimo, s.f.).

Vegetación. De acuerdo con Rzedowski (1965, 1978) los tipos de vegetación que se encuentran en el área de estudio son los siguientes: a) matorral desértico micrófilo. Se desarrolla en terrenos planos y en las partes inferiores de los cerros sobre sustratos de origen aluvial; se caracteriza por la predominancia de elementos arbustivos de hojas o folíolos pequeños como Larrea tridentata y Flourensia cernua. b) matorral desértico rosetófilo, que ocupa las laderas con substratos de roca sedimentaria; la fisonomía de esta comunidad se debe a la presencia de especies con hojas alargadas y estrechas, agrupadas en forma de roseta, pueden ser caulescentes como Yucca carnerosana o acaulescentes como Agave lechuguilla. c) matorral crasicaule que se desarrolla en laderas y planicies con substrato ígneo. Este tipo de vegetación se caracteriza por la dominancia fisonómica de cactáceas del género Opuntia. d) encinar arbustivo. Este tipo de vegetación se distingue por la dominancia, en el estrato superior, de especies del género Quercus. e) piñonar. Se desarrolla sobre suelos ígneos y sedimentarios. En la Sierra Monte Grande está caracterizado por la dominancia fisonómica total de Pinus discolor.

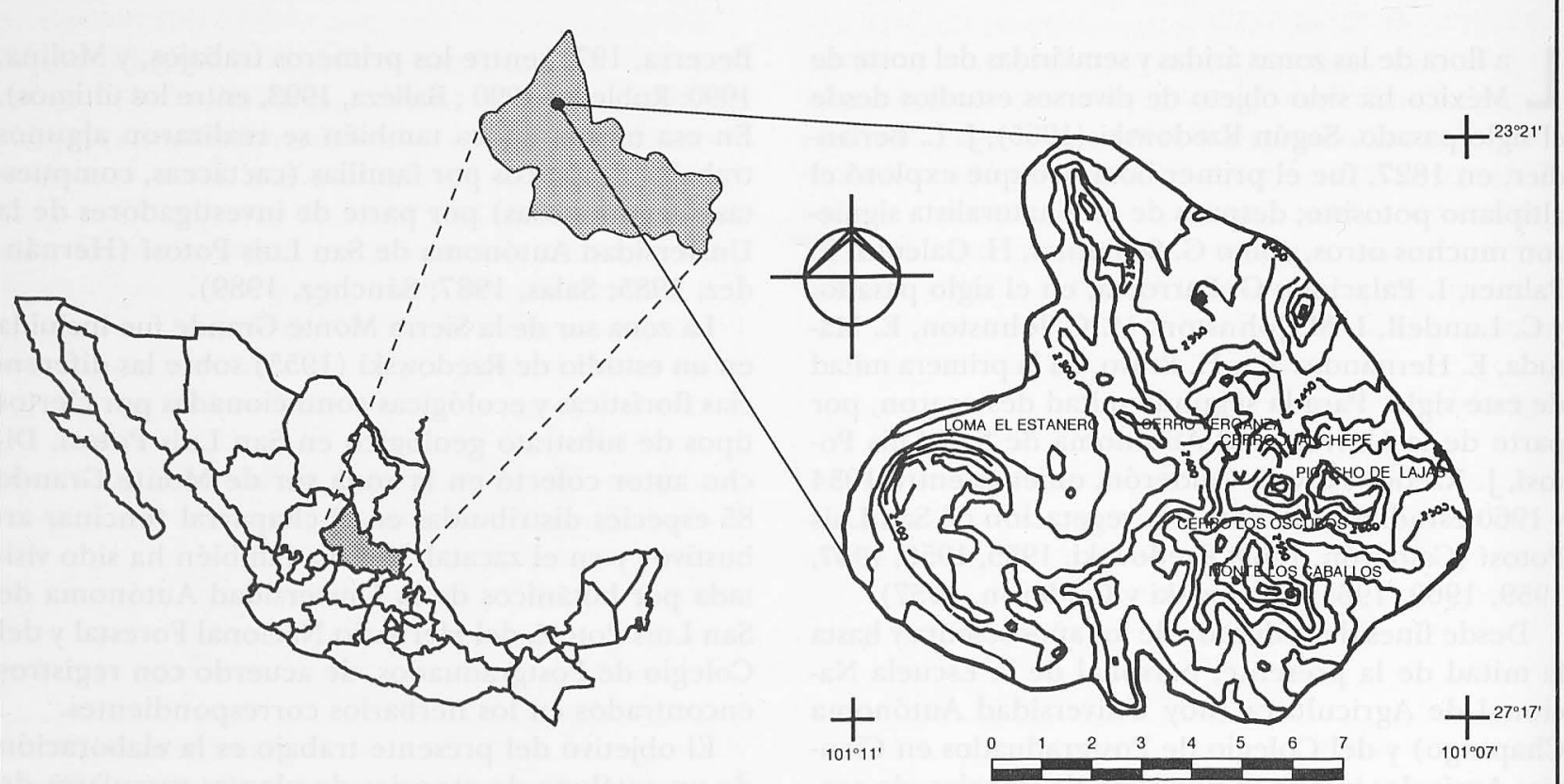

Figura 1. Localización y topografía de la Sierra Monte Grande, municipio de Charcas, S.L.P. 


\section{Métodos}

Se realizaron diez exploraciones botánicas entre agosto de 1989 y septiembre de 1990. Para asegurar una recolección próxima a la totalidad de las especies existentes, la última visita a la zona fue también de cotejo (J. R. Aguirre R., com. pers.). Se establecieron y siguieron ocho transectos de no menos de $1 \mathrm{~km}$ de longitud desde las partes altas hacia las bajas de la sierra. A cada $200 \mathrm{~m}$ se realizó un reconocimiento de las especies situadas desde ese punto hasta el alcance de la vista, en forma sectorial, hasta completar $360^{\circ}$, para verificar que todas las especies observadas habían sido ya recolectadas; si existía duda al respecto, se procedía a recolectarlas. Se procuró que todo el material botánico fuera lo más representativo posible de las características del individuo en particular y de la población en general, de acuerdo con Madrigal (1986). Para la identificación se recurrió a: 1) literatura botánica y actualizada, como revisiones y monografías sobre grupos taxonómicos y floras regionales; 2) el cotejo con los especímenes depositados en el Herbario-Hortorio (CHAPA) del Programa de Botánica; y 3) la ayuda de especialistas en diferentes grupos de plantas.

\section{Resultados y discusión}

En la Sierra Monte Grande se registraron 397 especies de plantas vasculares (lista 1) que pertenecen a 242 géneros de 74 familias (cuadro 2). Es difícil encontrar dos áreas que se puedan comparar florísticamente de una manera completa, pero para dar una idea de la cantidad de especies encontradas en Monte Grande se puede decir que Pinkava (1984) encontró en el bolsón de Cuatro Ciénegas, Coahuila un promedio de 0.43 especies $/ \mathrm{km}^{2}$ (860 especies en 2,000 $\mathrm{km}^{2}$; mientras que Villarreal (1994) registró en la sierra de la Paila, Coah. una relación de 0.41; en Guadalcázar, S.L.P. (Rzedowski, 1956) la relación fue de 0.70; en el Valle de San Luis Potosí fue de 0.30 (Calderón, 1960) y en La Amapola, S.L.P. fue de 0.85 (Luna et al., 1990) (cuadro 1). Las posibles razones para explicar la presencia de 9.49 especies por $\mathrm{km}^{2}$ en la Sierra Monte Grande son: a) el tamaño pequeño del área explorada; $b$ ) su localización en el extremo norte del altiplano potosino, en el sur de la altiplanicie septentrional y como una de las últimas estribaciones occidentales de la Sierra Madre Oriental; $c$ ) la existencia de una topografía accidentada, con substratos de rocas ígneas así como sedimentarias, en una región en la que predominan extensas planicies constituidas por material aluvial; $d$ ) la orientación predominantemente noroeste de las laderas de la sierra. Al parecer, todas estas características son favorables para que en Monte Grande se localicen cinco de los seis tipos de vegetación registrados para la zona árida de San Luis Potosí (sólo falta el zacatal) y zonas de ecotono en donde confluyen hasta cuatro tipos de vegetación.

Como se observa en el cuadro 2, la división Magnoliofita fue la mejor representada con $97 \%$ del total de especies encontradas (la clase Magnoliópsida tuvo $78.1 \%$ y la Liliópsida $18.9 \%$ ), las divisiones Polipodiofita y Licopodiofita representan, en conjunto, sólo el $2.25 \%$, y Pinofita el $0.75 \%$ de las especies.

En las asteráceas, poáceas, cactáceas y fabáceas se concentran $41.79 \%$ del total de las especies, mientras que en las 70 familias restantes se distribuye el $58.21 \%$ de los táxones específicos. La familia mejor representada es Asteraceae con $19.89 \%$ de las especies, seguida por Poaceae con $11.33 \%$ y Cactaceae con $6.04 \%$ (cuadro 3). Estas cifras coinciden con lo afirmado por Rzedowski (1991), quien señala que en el norte y centro del país están mejor representadas las compuestas, las gramíneas y las cactáceas. Con respecto a las asteráceas es necesario añadir que, según Rzedowski (1972), México es considerado el centro de diversidad de este grupo de plantas, y que existe una tendencia hacia el aumento de su número desde el sureste al noroeste de México; así, en los matorrales xerófi-

Cuadro 1. Comparación de la riqueza florística de la Sierra Monte Grande, con otras localidades de clima semiárido del centro-norte de México

\begin{tabular}{lcccl}
\hline Localidad & $\begin{array}{c}\text { Número de } \\
\text { especies }\end{array}$ & $\begin{array}{c}\text { Extensión } \\
\left(\mathbf{k m}^{2}\right)\end{array}$ & $\begin{array}{c}\text { Relación } \\
\text { especies/km }\end{array}$ & Referencia \\
\hline Bolsón de Cuatro Ciénegas, Coah. & 860 & 2,000 & 0.43 & Pinkava, 1984 \\
La Amapola, S.L.P. & 127 & 150 & 0.85 & Luna et al., 1990 \\
Guadalcázar, S.L.P. & $\pm 1,000$ & 1,500 & 0.70 & Rzedowski, 1956 \\
La Paila, Coah. & 712 & 1,700 & 0.41 & Villarreal Q., 1994 \\
Valle de San Luis Potosí, S.L.P. & 759 & 2,500 & 0.30 & Calderón, 1960 \\
Monte Grande, S.L.P. & 397 & 41.8 & 9.49 & Este trabajo \\
\hline
\end{tabular}


los, pastizales y bosques de Pinus-Quercus de la zona norte del país, es en donde se presenta una alta proporción de este grupo de plantas.

Cuadro 2. Números globales de los taxa de la flora vascular registrados en Monte Grande, S.L.P.

\begin{tabular}{lccc}
\hline & Familias & Géneros & Especies \\
\hline Lycopodiophyta & 1 & 1 & 1 \\
Polypodiophyta & 2 & 4 & 8 \\
Pinophyta & 3 & 3 & 3 \\
Magnoliophyta & & & \\
Magnoliopsida & 59 & 190 & 310 \\
$\quad$ Liliopsida & 9 & 46 & 76 \\
Total & 74 & 242 & 397 \\
\hline
\end{tabular}

El género con mayor número de especies fue Opuntia con 10, seguido por Salvia con ocho. Opuntia es un género nativo de América, muy bien representado en el norte de México (Bravo, 1978). En el altiplano potosino está presente como dominante fisonómico del matorral crasicaule, pero tiene especies en prácticamente todos los tipos de vegetación (algunas de forma exclusiva). Con respecto a Salvia, de 900 especies que existen en el mundo, 312 habitan en México (Ramamoorthy y Elliot, 1993), situación que se refleja en Monte Grande ya que sin ser Salvia dominante fisonómico de ningún tipo de vegetación, está presente en la mayor parte de ellos.

El $80 \%$ de las especies se localizaron en tres tipos de vegetación con hábitat de ladera: el matorral crasicaule $(30 \%)$, el matorral desértico rosetófilo (25\%) y el encinar arbustivo (25\%). Los atributos probablemente asociados a lo favorable de este tipo de ambiente para el desarrollo de plantas son: la frecuencia de las laderas con exposición al norte; la presencia de aguas rezumantes; y el hecho de que las laderas son sitios de paso y receptores (en microhábitats) de agua, suelo y nutrimentos que provienen de las partes más altas de la sierra. Lo anterior sin duda, favorece la abundancia de microhábitats adecuados para el desarrollo de una mayor diversidad de especies.

Rzedowski (1957; 1965) y Marroquín et al. (1981) coinciden en que el matorral crasicaule recibe, en general, mayor cantidad de lluvia respecto a otras comunidades xerófilas y que señala el paso hacia regiones más húmedas. Este tipo de vegetación tiene mayor riqueza de especies que otros matorrales xerófilos, como el micrófilo y el rosetófilo (Rzedowski, 1965; Del Castillo, 1982; Juárez et al., en prensa).Las características edáficas asociadas al matorral desértico rosetófilo como el segundo tipo de vegetación con mayor riqueza florística son, según Aldrete y Aguirre (1981): alta proporción de pedregosidad, de mantillo y/o de cubierta vegetal, suelos oscuros, poco alcalinos y ricos en materia orgánica.

El encinar arbustivo es un tipo de vegetación intermedio entre los matorrales xerófilos de clima seco y los pinares y encinares de clima submésico (Rzedowski, 1965). En efecto, en el área de estudio, el encinar arbustivo es favorecido por la frecuente aparición de niebla, lo que aunado a las características de la cubierta vegetal que se desarrolla en laderas, mencionadas en párrafos anteriores, favorece la presencia de diversas especies.

La baja cantidad de especies encontradas en el matorral micrófilo (11\% del total de las especies) puede deberse a que es un tipo de vegetación que se desarrolla en climas muy áridos, en terrenos planos, con baja pedregosidad, poca materia orgánica y alta proporción de suelo sin cubierta vegetal o sin mantillo (Rzedowski, 1965; Aldrete y Aguirre, 1981), lo que genera un hábitat pobre y homogéneo, poco propicio para una mayor diversidad de plantas.

A pesar de tener en gran medida las características climáticas del encinar arbustivo y de localizarse en laderas, el piñonar ha sido considerado por Passini (1982) como un tipo de vegetación de poca riqueza de especies. Para el caso de Monte Grande, hay que añadir que es el único tipo de vegetación sometido a intenso pastoreo por parte de ganado caprino, se localiza mayormente en laderas con exposición sur y, a diferencia del encinar arbustivo, no se ve favorecido por las neblinas frecuentes.

Nuevos registros floristicos. Los nuevos registros para el altiplano potosino y probablemente para el zacatecano, se dividieron en:

a) Nuevos registros significativos. Son las especies que por la distribución registrada en la literatura, resulta extraordinario encontrarlas en San Luis Potosí y de esa forma se amplía significativamente su área: Arabis fendleri, Arracacia nelsonii, Castilleja integra, Cercocarpus montanus, Cirsium rhaphilepis, Coryphantha

Cuadro 3. Familias con mayor número de especies en la flora vascular de Monte Grande, S.L.P.

\begin{tabular}{lccc}
\hline Familias & Géneros & \multicolumn{2}{c}{ Especies } \\
\cline { 3 - 4 } & & Número & $\begin{array}{c}\text { Porcentaje } \\
\text { del total }\end{array}$ \\
\hline Asteraceae & 45 & 79 & 19.89 \\
Poaceae & 25 & 45 & 11.33 \\
Cactaceae & 10 & 24 & 6.04 \\
Fabaceae & 9 & 18 & 4.53 \\
\hline
\end{tabular}


villarensis, Grindelia inuloides, Holodiscus dumosus, Juniperus erythrocarpa, Linum lewisii, Microsechium helleri, Nolina texana, Opuntia chaffeyi, Phoradendron villosum, Physalis caudella, Seymeria scabra, Stipa neomexicana, Streptanthus hyacinthoides, Teucrium laciniatum, Viguiera rhombifolia y Villadia cucullata (21 en total).

b) Nuevos registros poco significativos. Son las especies que en función de la distribución conocida era muy probable que se encontraran en la parte árida de San Luis Potosí: Arracacia atropurpurea, Cologania angustifolia, Condalia spathulata, Erysimum capitatum, Erigeron janivultus, E. karvinskianus, Hieracium abscissum, H. dysonimum, H. pringlei, Ipomopsis pinnata ${ }_{2}$ Juncus tenuis, Lithospermum viride, Oenothera kunthiana, Oxalis jacquiniana, Parietaria pensylvanica, Pennellia longifolia, Penstemon roseus, Prunus microphylla, Psoralea rhombifolia, Quercus depressipes, Sedum greggii, Spiranthes cinnabarina, Thelypodium wrightii y Tillandsia erubescens (24 en total).

\section{Agradecimientos}

Los autores desean expresar su gratitud al Dr. J. R. Aguirre R. por sus valiosos consejos, al Dr. E. García M. por su apoyo para la exploración botánica, al Biól. S. Zamudio por la cuidadosa revisión del escrito. A los expertos que auxiliaron en la identificación o confirmación de las especies: B. L. Turner y G. Nesom, para la familia Asteraceae; M. González, para la familia Poaceae; M. Luna, familia Solanaceae; S. Arias y A. Zimmerman, familia Cactaceae; M. C. Herrera, pteridofitas; J. Pérez de la R., género Juniperus; A. García, Dasylirion y Nolina; J. Marroquín, género Berberis; A. Ávila, género Pinus; F. Zavala, género Quercus.

Lista 1. Plantas vasculares de la Sierra de Monte Grande, Mpio. de Charcas, S.L.P. (Las categorías superiores están escritas con base en Cronquist (1981) y en Tryon y Tryon (1982); las familias, los géneros y las especies están ordenadas alfabéticamente; los nombres de los autores están escritos con base en Brummitt y Powell (1992).

FAMILIAS Clave*
Especies

\section{LYCOPODIOPHYTA}

SELAGINELLACEAE

Selaginella sartorii Hieron.

$\mathrm{Hp} / \mathrm{C} / 962$

\section{POLYPODIOPHYTA}

\section{POLYPODIACEAE}

Polypodium guttatum Maxon

Polypodium thyssanolepis

A. Braun ex Klotzsch

Hp/P-E,R,E/1129

$\mathrm{Hp} / \mathrm{C} / 969$

\section{PTERIDACEAE}

Adiantum capillus-veneris L.

Hp/R-C/906

Cheilanthes bonariensis (Willd.) Proctor Hp/R,P-E/1075

Cheilanthes sinuata

(Lag. ex Sw.) Domin

Pellaea cordifolia

(Sessé \& Moc.) A. R. Sm.

Pellaea sagittata (Cav.) Link

Pellaea ternifolia (Cav.) Link

$\mathrm{Hp} / \mathrm{R} / 1112$

$\mathrm{Hp} / \mathrm{C} / 1121$

$\mathrm{Hp} / \mathrm{R} / 947$

$\mathrm{Hp} / \mathrm{R}, \mathrm{P}-\mathrm{E} / 1079$

\section{PINOPHYTA}

CUPRESSACEAE

Juniperus erythrocarpa Cory

Arb/M-R/901

\section{EPHEDRACEAE}

Ephedra aspera Engelm.

Arb/M-R/1051

PINACEAE

Pinus discolor Bailey \& Hawksw.
FAMILIAS

Especies

Clave*

MAGNOLIOPHYTA

MAGNOLIOPSIDA

ACANTHACEAE

Dyschoriste decumbens

(A. Gray) Kuntze.

Stenandrium dulce (Cav.) Nees

$\mathrm{Hp} / \mathrm{M} / 674$

$\mathrm{Hp} / \mathrm{E} / 934$

AMARANTHACEAE

Alternanthera caracasana H.B.K.

Amaranthus hybridus $\mathrm{L}$.

$\mathrm{Hp} / \mathrm{C} / 912$

$\mathrm{Ha} / \mathrm{M}, \mathrm{C}, \mathrm{E} / 758$

ANACARDIACEAE

Rhus microphylla Engelm.

Rhus trilobata Nutt.

Schinus molle L.

Arb/C,M/1017

Arb/R/837

$\mathrm{Ar} / \mathrm{C} / 1052$

\section{APIACEAE (UMBELLIFERAE)}

Arracacia atropurpurea

(Lehm.) Benth. \& Hook.

Arracacia nelsoni Coult. \& Rose

Eryngium carlinae Delar $\mathrm{f}$.

Eryngium serratum Cav.

$\mathrm{Hp} / \mathrm{E}, \mathrm{R} / 248$

$\mathrm{Hp} / \mathrm{R} / 826$

$\mathrm{Hp} / \mathrm{C}, \mathrm{E}-\mathrm{P} / 658$

$\mathrm{Hp} / \mathrm{E} / 821$

ASCLEPIADACEAE

Asclepias linaria Cav.

Suf/C/437

Metastelma angustifolium Turcz. 


\section{FAMILIAS}

Especies

Acourtia wrightii

(A. Gray) Reveal \& King

Ageratina petiolare

(DC.) King \& $\mathrm{H}$. Rob.

Ageratina scorodonioides

(A. Gray) King \& H. Rob.

Ageratum corymbosum Zucc. ex Pers.

Ambrosia confertiflora DC.

Aphanostephus ramosissimus DC.

Artemisia klotzschiana Besser

Artemisia ludoviciana Nutt.

Aster arenosus (Haller) Blake

Aster gymnocephalus (DC.) A. Gray

Aster subulatus Michx.

Baccharis pteronioides DC.

Bahia absinthifolia Benth.

Bahia schaffneri Wats.

Bidens odorata Cav.

Bidens schaffneri var. wrightii

(A. Gray) Turner \& Melchert

Brickellia laciniata A. Gray

Brickellia pedunculosa

(DC.) Harc. \& Beaman

Clave*

Brickellia veronicifolia (H.B.K.) A.

Cirsium rhaphilepis (Hemsl.) Petrak

Conyza bonariensis (L.) Cronquist

Conyza filaginoides (DC.) Hieron.

Chaptalia nutans (L.) Polak

Chrysactinia mexicana A. Gray

Dahlia dissecta Wats.

Dyssodia acerosa DC.

Dyssodia papposa (Vent.) Hitchc.

Dyssodia pentachaeta (DC.) Rob.

Dyssodia pinnata (Cav.) Rob.

Dyssodia setifolia (Lag.) Rob.

Erigeron calcicola Greenm.

Erigeron flagellaris A. Gray

Erigeron janivultus Nesom

Erigeron karvinskianus DC.

Flourensia cernua DC.

Gaillardia comosa A. Gray

Galinsoga parviflora Cav.

Gnaphalium brachypterum DC.

Gnaphalium roseum H.B.K.

Gnaphalium semiamplexicaule DC:

Grindelia inuloides Willd.

Grindelia oxylepis Greene

Grindelia subdecurrens DC.

Gymnosperma glutinosum (Spreng.) Less.

Haplopappus spinulosus (Pursh) DC.
Arb/M,R/907

Suf/R/1034

Suf/M,C,M-R,

E/984

Arb/M-R,E,P/669

$\mathrm{Hp} / \mathrm{C} / 681$

$\mathrm{Hp} / \mathrm{M} / 619$

$\mathrm{Hp} / \mathrm{C} / 966$

$\mathrm{Hp} / \mathrm{C}, \mathrm{M}-\mathrm{R} / 980$

$\mathrm{Hp} / \mathrm{C}, \mathrm{M}, \mathrm{M}-\mathrm{R}$,

P-E-R/876

$\mathrm{Hp} / \mathrm{E} / 748$

$\mathrm{Ha} / \mathrm{C} / 966$

$\mathrm{Arb} / \mathrm{E} / 1029$

$\mathrm{Hp} / \mathrm{C}, \mathrm{M}, \mathrm{E}-\mathrm{P} / 657$

$\mathrm{Hp} / \mathrm{P}, \mathrm{C} / 987$

$\mathrm{Ha} / \mathrm{C}, \mathrm{P}-\mathrm{E}-\mathrm{R}$,

E-P/877

$\mathrm{Hp} / \mathrm{E} / 257$

$\mathrm{Arb} / \mathrm{R} / 478$

$\mathrm{Ha} / \mathrm{E} / 822$

Suf/R,C,E/431

$\mathrm{Hp} / \mathrm{M}-\mathrm{R} / 554$

$\mathrm{Ha} / \mathrm{C}, \mathrm{M}-\mathrm{R} / 978$

$\mathrm{Ha} / \mathrm{C}, \mathrm{E}, \mathrm{E}-\mathrm{P} / 760$

$\mathrm{Hp} / \mathrm{R} / 502$

$\mathrm{Arb} / \mathrm{R}, \mathrm{E} / 434$

$\mathrm{Hp} / \mathrm{R} / 808$

$\mathrm{Hp} / \mathrm{M}-\mathrm{R} / 840$

$\mathrm{Ha} / \mathrm{E} / 766$

$\mathrm{Hp} / \mathrm{C}, \mathrm{E}-\mathrm{P} / 947$

$\mathrm{Hp} / \mathrm{R}, \mathrm{C}, \mathrm{P}, \mathrm{E}$,

E-P/581

$\mathrm{Hp} / \mathrm{R}, \mathrm{C}, \mathrm{E} / 322$

$\mathrm{Ha} / \mathrm{M}-\mathrm{C} / 714$

$\mathrm{Hp} / \mathrm{C}, \mathrm{E}, \mathrm{E}-\mathrm{P} / 500$

$\mathrm{Hp} / \mathrm{R} / 801 \mathrm{~b}$

$\mathrm{Hp} / \mathrm{C} / 899$

Arb/M,M-R/887

$\mathrm{Hp} / \mathrm{R}, \mathrm{M} / 405$

$\mathrm{Ha} / \mathrm{C}, \mathrm{P}, \mathrm{P}-\mathrm{E}-\mathrm{R} / 634$

$\mathrm{Hp} / \mathrm{R}, \mathrm{E} / 945$

$\mathrm{Hp} / \mathrm{E}, \mathrm{R} / 1134$

$\mathrm{Hp} / \mathrm{C} / 705$

$\mathrm{Hp} / \mathrm{R} / 449$

$\mathrm{Hp} / \mathrm{C}, \mathrm{E} / 780$

$\mathrm{Hp} / \mathrm{C}, \mathrm{E} / 267$

Suf/M,E/843

$\mathrm{Hp} / \mathrm{C}, \mathrm{M}, \mathrm{R}$,

$M-R M-C / 1023$
FAMILIAS

Especies

Clave* $^{*}$

Heterosperma pinnatum Cav.

Hieracium abscissum Less.

Hieracium dysonymum Blake

Hieracium pringlei A. Gray

Kuhnia chlorolepis Woot. \& Standl.

Laennecia sophiifolia

(Kunth) Nesom

Machaeranthera crutchfieldi Turner

Machaeranthera pinnatifida

var. chihuahuana Turner \& Hartm.

Parthenium argentatum A. Gray

Parthenium bipinnatifidum

(Ort.) Rollins

Parthenium incanum H.B.K.

Perymenium mendezii DC.

Pinaropappus roseus (Less.) Less.

Porophyllum tagetoides

(H.B.K.) DC.

Senecio stoechadiformis DC.

Solidago velutina DC.

Sonchus oleraceus L.

Stevia eupatoria (Spreng.) Willd.

Stevia salicifolia Cav.

Stevia tephra Rob.

Tagetes lucida Cav.

Tagetes micrantha Cav.

Taraxacum officinale Weber

Thelesperma simplicifolium A. Gray

Townsendia mexicana A. Gray

Viguiera cordifolia A. Gray

Viguiera linearis (Cav.) Sch. Bip.

Viguiera multiflora (Nutt.) Blake

Viguiera rhombifolia

(Rob. \& Greenm.) Blake

Zaluzania mollisima A. Gray

Zaluzania triloba (Ort.) Pers.

Zinnia acerosa (DC.) A. Gray

Zinnia juniperifolia (DC.) A. Gray

\section{BERBERIDACEAE}

Berberis pinifolia (Lundell) C.H. Mull.

Berberis trifoliolata Moric.

$\mathrm{Ha} / \mathrm{C}, \mathrm{E}, \mathrm{P} / 610$

$\mathrm{Hp} / \mathrm{E} / 382$

$\mathrm{Hp} / \mathrm{E} / 393$

$\mathrm{Ha} / \mathrm{E} / 269$

$\mathrm{Hp} / \mathrm{R} / 801$

$\mathrm{Ha} / \mathrm{C}, \mathrm{E}-\mathrm{P} / 960$

$\mathrm{Hp} / \mathrm{R} / 932$

Hp/M-R,C/1970

Arb/M-R/847

$\mathrm{Ha} / \mathrm{C} / 562$

Arb/M,R/ 312

$\mathrm{Hp} / \mathrm{R}, \mathrm{E}, \mathrm{P} / 608$

$\mathrm{HP} / \mathrm{R}, \mathrm{M}, \mathrm{C}$,

$\mathrm{E}-\mathrm{P} / 527$

$\mathrm{Hp} / \mathrm{R} / 838$

$\mathrm{Hp} / \mathrm{R} / 1138$

$\mathrm{Hp} / \mathrm{R}, \mathrm{R}-\mathrm{M}$,

$M-C / 897$

$\mathrm{Hp} / \mathrm{P}-\mathrm{E}-\mathrm{R} / 871$

$\mathrm{Hp} / \mathrm{E} / 767$

$\mathrm{Hp} / \mathrm{R}, \mathrm{C} / 621$

$\mathrm{Hp} / \mathrm{R} / 834$

$\mathrm{Ha} / \mathrm{P} / 1130$

$\mathrm{Ha} / \mathrm{P} / 965$

$\mathrm{Hp} / \mathrm{C} / 988$

$\mathrm{Hp} / \mathrm{E} / 383$

$\mathrm{Hp} / \mathrm{C}, \mathrm{R} / 996$

Suf/E,C,M-R/893

$\mathrm{Hp} / \mathrm{C}, \mathrm{R} / 827$

$\mathrm{Hp} / \mathrm{R} / 933$

$\mathrm{Hp} / \mathrm{R} / 285$

$\mathrm{Arb} / \mathrm{C} / 721$

$\mathrm{Hp} / \mathrm{R} / 790$

$\mathrm{Hp} / \mathrm{M}, \mathrm{R} / 894$

$\mathrm{Hp} / \mathrm{R} / 448$

\section{BORAGINACEAE}

Cryptantha albida (H.B.K.) I. M. Johnst.

Lithospermum viride Greene

$\mathrm{Hp} / \mathrm{R}-\mathrm{M} / 1177$

\section{BRASSICACEAE (CRUCIFERAE)}

Arabis fendleri (Wats.) Greene

Asta schaffneri (Wats.) Schulz

Eruca sativa Mill.

Erysimum capitatum (Dougl.) Greene

Halimolobos palmeri (Hemsl.) Schulz
Suf/C,R/833

$\mathrm{Arb} / \mathrm{C} / 993$

$\mathrm{Arb} / \mathrm{C} / 1057$

$\mathrm{Bi} / \mathrm{E} / 1156$

$\mathrm{Hp} / \mathrm{R}, \mathrm{E} / 1152$

$\mathrm{Ha} / \mathrm{E}, \mathrm{R} / 1174$

$\mathrm{Bi} / \mathrm{R} / 284$

$\mathrm{Bi} / \mathrm{C} / 574$ 


\section{FAMILIAS}

Especies

Halimolobos polysperma (Fourn.) Schulz Lepidium virginicum $\mathrm{L}$.

Lesquerella argyraea (A. Gray) Wats.

Lesquerella purpurea (A. Gray) Wats.

Nerisyrenia camporum (A. Gray) Greene Pennellia longifolia (Benth.) Rollins

Streptanthus hyacinthoides Hook.

Thelypodium wrightii A. Gray

\section{CACTACEAE}

Ariocarpus retusus Scheidw.

$\mathrm{Cra} / \mathrm{R}-\mathrm{M} / 1147$

Coryphantha radians (DC.) Britton \& Rose Cra/R/1114

Coryphantha villarensis Backeb.

$\mathrm{Cra} / \mathrm{E} / 1144$

Echinocactus horizonthalonius Lem.

Echinocactus platyacanthus Link \& Otto

Echinocereus cinerascens (DC.) Lem.

Echinocereus pectinatus

(Scheidw.) Engelm.

Echinofossulocactus dichroacanthus

(Mart.) Britton \& Rose

Ferocactus latispinus (Haw.) Britton \& Rose

Ferocactus pilosus (Gal.) Werderm.

Cra/R-M/1178

$\mathrm{Cra} / \mathrm{R} / 1064$

$\mathrm{Cra} / \mathrm{C} / 1180$

\section{$\mathrm{Cra} / \mathrm{R}, \mathrm{E} / 1036$}

$\mathrm{Cra} / \mathrm{E} / 1000$

$\mathrm{Cra} / \mathrm{C} / 1163$

$\mathrm{Cra} / \mathrm{R} / 1045$

Glandulicactus uncinatus (Gal.) Backeb. Cra/R-C/1148

Mammillaria formosa Gal.

Mammillaria uncinata Zucc.

Neolloydia conoidea

(DC.) Britton \& Rose

Opuntia cantabrigiensis Lynch

Opuntia chaffeyi Britton \& Rose

Opuntia imbricata (Haw.) DC.

Opuntia leucotricha DC.

Opuntia microdasys (Lehm.) Pfeiff.

Opuntia rastrera Weber

Opuntia robusta Wendl.

Opuntia stenopetala Engelm.

Opuntia streptacantha Lem.

Opuntia tunicata (Lehm.) Link \& Otto

$\mathrm{Cra} / \mathrm{R}, \mathrm{C}-\mathrm{R} / 1164$

$\mathrm{Cra} / \mathrm{E} / 1106$

$\mathrm{Cra} / \mathrm{R} / 1049$

$\mathrm{Cra} / \mathrm{C} / 1167$

$\mathrm{Cra} / \mathrm{R} / 1146$

$\mathrm{Cra} / \mathrm{R}, \mathrm{C} / 1048$

$\mathrm{Cra} / \mathrm{C} / 1022$

$\mathrm{Cra} / \mathrm{R} / 1049$

$\mathrm{Cra} / \mathrm{C}-\mathrm{R} / 1165$

$\mathrm{Cra} / \mathrm{C} / 1021$

$\mathrm{Cra} / \mathrm{R} / 1027$

$\mathrm{Cra} / \mathrm{C} / 1166$

$\mathrm{Cra} / \mathrm{R} / 1050$

CAESALPINIACEAE (LEGUMINOSAE, en parte)

Hoffmanseggia glauca (Ort.) Eifert Hp/C/566

Senna bauhinioides (A. Gray)

Irwin \& Barneby

Senna crotalarioides

(Kunth) Irwin \& Barneby

Hp/C,C-M/1119

$\mathrm{Hp} / \mathrm{E}, \mathrm{M}-\mathrm{C} / 1097$

\section{CAPRIFOLIACEAE}

Lonicera pilosa (H.B.K.) Willd.

Trep/E/923

\section{CARYOPHYLLACEAE}

Arenaria lycopodioides

Willd. ex Schlecht.

Corrigiola andina Triana \& Planch.

Drymaria arenarioides Willd.

Drymaria glandulosa Bartl.

\section{FAMILIAS}

Especies

Clave*

Drymaria gracilis Cham. \& Schlecht.

$\mathrm{Ha} / \mathrm{P}, \mathrm{E}, \mathrm{C} / 1058$

\section{CELASTRACEAE}

Mortonia palmeri Hemsl.

$\operatorname{Arb} / \mathrm{R}, \mathrm{E} / 1028$

\section{CHENOPODIACEAE}

Chenopodium graveolens Willd.

Chenopodium murale L.

Salsola kali L.

$\mathrm{Ha} / \mathrm{C}, \mathrm{M}-\mathrm{R}, \mathrm{E} / 842$

$\mathrm{Ha} / \mathrm{M} / 982$

$\mathrm{Ha} / \mathrm{M} / 538$

\section{CISTACEAE}

Helianthemum glomeratum Lag.

Helianthemum patens Hemsl.

$\mathrm{Hp} / \mathrm{P} / 1001 \mathrm{a}$

$\mathrm{Hp} / \mathrm{E}, \mathrm{P}, \mathrm{C}$,

E-P/1012

\section{CONVOLVULACEAE}

Dichondra argentea Humb. \& Bonpl.

Evolvulus sericeus Sw.

Ipomoea capillacea G. Don

Ipomoea pubescens Lam.

\section{Ras/R,R-M, $\mathrm{P} / 1088$}

$\mathrm{Hp} / \mathrm{P}, \mathrm{C} / 594$

$\mathrm{Hp} / \mathrm{E}, \mathrm{R} / 419$

Hp/P-E-R,E-M, E-P/869

\section{CRASSULACEAE}

Echeveria mucronata (Bak.) Schlecht.

Sedum greggii Hemsl.

Villadia cucullata Rose

Villadia parviflora (Hemsl.) Rose

\section{$\mathrm{Cra} / \mathrm{E}, \mathrm{P} / 1138$ \\ $\mathrm{Hp} / \mathrm{R} / 1065$ \\ $\mathrm{Cra} / \mathrm{R} / 1175$ \\ Cra/E,E-P/1131}

\section{CUCURBITACEAE}

Apodanthera undulata A. Gray

Cucurbita foetidissima H.B.K.

Microsechium helleri (Peyr.) Cogn.

Ras/C/1120

Ras/M,P-E/1083

Ras/C,C-M/957

\section{ERICACEAE}

Arbutus xalapensis H.B.K.

Arctostaphylos pungens H.B.K.

Sar/E/1032

Sar, Arb/E/1062

\section{EUPHORBIACEAE}

Acalypha phleoides Cav.

Croton pottsii (Klotzsch) Muell.

Euphorbia furcillata H.B.K.

Euphorbia hirta L.

Euphorbia macropus

(Klotzsch \& Garcke) Boiss.

Euphorbia serpyllifolia Pers.

Jatropha dioica Sessé ex Cerv.

Tragia nepetifolia Cav.

$\mathrm{Hp} / \mathrm{E}, \mathrm{R}, \mathrm{C}-\mathrm{R}$, $M-R / 474$

Arb/R/1170

Arb/E/1041

$\mathrm{Ha} / \mathrm{M}-\mathrm{R} / 525$

$\mathrm{HP} / \mathrm{R}, \mathrm{R}-\mathrm{E} / 470$

Ras/C,R/1111

Arb/C/1056

Hp/R-E,M-R/916

FABACEAE (LEGUMINOSAE, en parte) Astragalus coriaceus Hemsl.

Astragalus diphacus Wats.

Astragalus hypoleucus Schaw.

Astragalus mollissimus Torr.
$\mathrm{Hp} / \mathrm{R}, \mathrm{M}-\mathrm{R} / 1161$

$\mathrm{Hp} / \mathrm{R}, \mathrm{E}, \mathrm{M}-\mathrm{R} / 881$

$\mathrm{Hp} / \mathrm{E}, \mathrm{R} / 425$

$\mathrm{Hp} / \mathrm{M} / 741$ 
FAMILIAS

Especies

Astragalus wootoni (Kunth) Barneby

Calia secundiflora (Ort.) Yakovi

Cologania angustifolia Kunth

Dalea bicolor Humb. \& Bonpl.

Dalea brachystachys A. Gray

Dalea capitata Wats.

Dalea foliolosa (Ait.) Barneby

Dalea lutea (Cav.) Willd.

Dalea pogonathera A. Gray

Lathyrus parvifolius Wats.

Phaseolus vulgaris $\mathrm{L}$.

Psoralea rhombifolia Torr. \& A. Gray

Trifolium amabile H.B.K.

Vicia pulchella H.B.K.

\section{FAGACEAE}

Quercus cordifolia Trel.

Quercus depressipes Trel.

Quercus eduardii Trel.

Quercus grisea Liebm.

Quercus potosina Trel.

Quercus repanda Humb. \& Bonpl.

Quercus rugosa Née

\section{GARRYACEAE}

Garrya lindheimeri Torr.

Garrya ovata Benth.

\section{GER.ANIACEAE}

Erodium cicutarium (L.) L'Hér.

\section{HYDROPHYLLACEAE}

Nama biflorum Choisy

Nama dichotomum

(Ruiz \& Pavón) Choisy

Nama palmeri A. Gray ex Hemsl.

Nama undulatum H.B.K.

\section{KRAMERIACEAE}

Krameria lanceolata Torr.

LAMIACEAE (LABIATAE)

Hedeoma plicatum Torr.

Poliomintha longiflora A. Gray

Salvia axillaris Moc. \& Sessé

Salvia ballotaeflora Benth.

Salvia chamaedryoides Cav.

Salvia microphylla H.B.K.

Salvia nana H.B.K.

Salvia prunelloides H.B.K.
Clave*

$\mathrm{Hp} / \mathrm{C} / 995$

$\mathrm{Arb} / \mathrm{R}, \mathrm{E}, \mathrm{M}-\mathrm{R} / 911$

Ras/E/253

Arb/M,R,C/565

$\mathrm{Arb} / \mathrm{R} / 319$

$\mathrm{Arb} / \mathrm{C}, \mathrm{R} / 680$

$\mathrm{Hp} / \mathrm{C}, \mathrm{E} / 1004$

Arb/R,M-R,E/892

$\mathrm{Hp} / \mathrm{M} / 677$

Tre/R,E/1137

$\mathrm{Ha}^{*} / \mathrm{M}-\mathrm{R} / 841$

$\mathrm{Hp} / \mathrm{R} / 811$

$\mathrm{Hp} / \mathrm{C} / 729$

Trep/E/1136

$\mathrm{Ar} / \mathrm{P} / \mathrm{P} 182$

Sar/P/1115

Arb/P,E/1117

$\mathrm{Ar} / \mathrm{P} / 1053$

Arb/E,E-P/1107

Arb/E,C-R/1158

$\mathrm{Ar} / \mathrm{E} / 1159$

$\operatorname{Arb} / \mathrm{R} / 471$

$\mathrm{Arb} / \mathrm{R}, \mathrm{E} / 117$

$\mathrm{Ha} / \mathrm{R}, \mathrm{C}, \mathrm{P}-\mathrm{E}-\mathrm{R}$, R-M,P-E/712

$\mathrm{Hp} / \mathrm{E}, \mathrm{C} / 445$

$\mathrm{Ha} / \mathrm{C} / 727$

$\mathrm{Hp} / \mathrm{M}, \mathrm{M}-\mathrm{R}$,

C-R,E/1094

$\mathrm{Hp} / \mathrm{M}-\mathrm{R} / 971$

$\mathrm{Hp} / \mathrm{E} / 436$

$\mathrm{Hp} / \mathrm{R} / 799$

$\mathrm{Arb} / \mathrm{M}, \mathrm{C}, \mathrm{R}, / 731$

$\mathrm{Hp} / \mathrm{C}, \mathrm{P}, \mathrm{R}-\mathrm{E}$,

$P-E / 447$

Arb/M,C,R/731

Arb/P,R,C,

$\mathrm{P}-\mathrm{E}, / 1090$

Arb/E,R,M/765

$\mathrm{Hp} / \mathrm{P}, \mathrm{R}-\mathrm{E}, \mathrm{E}-\mathrm{P} / 1089$

$\mathrm{Hp} / \mathrm{E}, \mathrm{R}-\mathrm{E} / 832$
FAMILIAS

Especies

Clave*

Salvia reflexa Hornem.

$\mathrm{Ha} / \mathrm{M} / 309$

Salvia tiliifolia Vahl

Scutellaria potosina Brand.

Stachys nepetifolia Desf.

Teucrium cubense Jacq.

Teucrium laciniatum Torr.

$\mathrm{Ha} / \mathrm{C} / 733$

$\mathrm{HP} / \mathrm{R} / 815$

$\mathrm{Hp} / \mathrm{R}, \mathrm{C}, \mathrm{M}-\mathrm{R}$,

E/631

$\mathrm{Hp} / \mathrm{M}-\mathrm{R} / 523$

$\mathrm{HP} / \mathrm{E}, \mathrm{P}-\mathrm{E}-\mathrm{R} / 878$

\section{LINACEAE}

Linum flagellare (Small) Winkl.

Linum lewisii Pursh

Linum rupestre (A. Gray) Engelm.

$\mathrm{Hp} / \mathrm{C} / 923$

$\mathrm{Hp} / \mathrm{E}, \mathrm{M}-\mathrm{R} / 536$

$\mathrm{HP} / \mathrm{R}, \mathrm{C}, \mathrm{P}-\mathrm{E}-\mathrm{R}$,

$\mathrm{P}-\mathrm{E} / 42$

\section{LOASACEAE}

Mentzelia hispida Willd.

$\mathrm{Hp} / \mathrm{R} / 791$

\section{LOGANIACEAE}

Buddleia cordata H.B.K.

Buddleia scordioides H.B.K.

$\mathrm{Ar} / \mathrm{P}-\mathrm{E}-\mathrm{R} / 882$

$\mathrm{Arb} / \mathrm{C}, \mathrm{R} / 561$

LORANTHACEAE

Phoradendron schumannii Trel.

Phoradendron villosum Nutt.

$\mathrm{Epp} / \mathrm{E}, \mathrm{P} / 300 \mathrm{~b}$

$\mathrm{Epp} / \mathrm{E} / 300 \mathrm{a}$

\section{MALVACEAE}

Allowissadula floribunda

(Schlecht.) Fryxell

Malva parviflora $\mathrm{L}$.

Sida abutifolia Mill.

Sida rzedowskii Fryxell

Sphaeralcea angustifolia (Cav.) G. Don

Suf/C/956

$\mathrm{Ha} / \mathrm{M}-\mathrm{R} / 915$

$\mathrm{Hp} / \mathrm{C} / 555$

$\mathrm{Hp} / \mathrm{M}, \mathrm{P} / 616$

$\mathrm{Hp} / \mathrm{C}, \mathrm{E} / 1163$

MIMOSACEAE (LEGUMINOSAE, en parte)

Acacia schaffneri (Wats.) Herm.

Desmanthus virgatus (L.) Willd.

$\mathrm{Sar} / \mathrm{C} / 1183$

Havardia elachistophylla

(A. Gray ex Wats.) L. Rico

Havardia leptophylla (Cav.) L. Rico

Mimosa biuncifera Benth.

Mimosa zygophylla Benth.

Prosopis laevigata (Humb. \& Bonpl.

ex Willd.) M.C. Johnst.

$\mathrm{Hp} / \mathrm{P}-\mathrm{E}-\mathrm{R} / 883$

Arb/E/431

Arb/R/825

Arb/M,C/1184

Arb/R/468

Sar/C/1118

NYCTAGINACEAE

Cyphomeris gypsophiloides

(Mart. \& Gal.) Standl.

$\mathrm{Hp} / \mathrm{C}, \mathrm{C}-\mathrm{R} / 921$

Mirabilis glabrifolia (Ort.) I.M. Johnst. Mirabilis multiflora (Torr.) A. Gray

$\mathrm{Hp} / \mathrm{R}, \mathrm{C}, \mathrm{M}-\mathrm{R} / 700$

$\mathrm{Hp} / \mathrm{R} / 1168$

\section{OLEACEAE}

Menodora helianthemoides

Humb. \& Bonpl. 


\section{FAMILIAS}

Especies

\section{ONAGRACEAE}

Gaura coccinea Nutt. ex Pursh Oenothera kunthiana (Spach) Munz Oenothera pringlei (Munz) Munz Oenothera rosea L'Her. ex Ait.

\section{OXALIDACEAE}

Oxalis albicans H.B.K.

Oxalis decaphylla H.B.K.

Oxalis jacquiniana H.B.K.

\section{PAPAVERACEAE}

Argemone ochroleuca Sweet

\section{PHYTOL.ACCACEAE}

Phytolacca icosandra L.

\section{PLANTAGINACEAE}

Plantago nivea H.B.K.

\section{PLUMBAGINACEAE}

Plumbago pulchella Boiss.

\section{POLEMONIACEAE}

Gilia rigidula Benth.

Ipomopsis pinnata (Cav.) G. Don

Loeselia coerulea (Cav.) G. Don

\section{POLYGALACEAE}

Polygala berlandieri Wats.

Polygala compacta Rose

\section{POLYGONACEAE}

Eriogonum jamesii Benth.

Eriogonum wrightii Torr.

\section{PORTULACACEAE}

Paronychia mexicana Hemsl.

Portulaca pilosa L.

Talinum aurantiacum Engelm.

Talinum lineare H.B.K.

\section{RANUNCULACEAE}

Clematis drummondii Torr. \& A. Gray

\section{RESEDACEAE}

Reseda luteola L.

\section{RHAMNACEAE}

Ceanothus greggii A. Gray

Condalia spathulata A. Gray

\section{ROSACEAE}

Amelanchier denticulata (H.B.K.) Koch
$\mathrm{Ha} / \mathrm{M}, \mathrm{M}-\mathrm{R} / 890$

\section{Clave*}

$\mathrm{Hp} / \mathrm{R}, \mathrm{C}, \mathrm{M}-\mathrm{R} / 908$

$\mathrm{Hp} / \mathrm{R}, \mathrm{P}-\mathrm{R} / 874$

$H p / R, E, R-E / 388$

$\mathrm{Ha} / \mathrm{E}, \mathrm{E}-\mathrm{P}, \mathrm{R}$, C/630

$\mathrm{Hp} / \mathrm{E}, \mathrm{C} / 715$

$\mathrm{Hp} / \mathrm{R} / 1046$

$\mathrm{Ha} / \mathrm{M} / 307$

Hp/P-E/1082

Hp/P,C,P-E/666

$\mathrm{Hp} / \mathrm{C} / 961$

$\mathrm{Hp} / \mathrm{R}, \mathrm{E} / 444$

Hp/M-R,C/532

Hp/M-R,C/711

$\mathrm{Hp} / \mathrm{P}, \mathrm{R} / 622 \mathrm{~b}$

$\mathrm{Hp} / \mathrm{C} / 723$

Hp/E,R,P,E-R/1085

Suf/M,C/1085

$\mathrm{Hp} / \mathrm{C} / 950 \mathrm{a}$

$\mathrm{Hp} / \mathrm{C}, \mathrm{E}-\mathrm{P} / 673$

$\mathrm{Hp} / \mathrm{P}-\mathrm{E} / 352$

$\mathrm{Hp} / \mathrm{C} / 510$

Esc/M, R/796

E, R/367

Arb/M/417
$\mathrm{Hp} / \mathrm{P}-\mathrm{E} / 1081$
FAMILIAS

Especies

Clave*

Cercocarpus montanus Raf.

Cowania plicata D. Don

Holodiscus dumosus Heller

Lindleya mespiloides

(H.B.K.) Rydb.

Prunus microphylla (H.B.K.) Hemsl.

Arb/R/1172

Arb/R,R-M/902

Arb/E/1110

Arb/R,M/1047

RUBIACEAE

Bouvardia ternifolia (Cav.) Schlecht. Hedyotis rubra (Cav.) A. Gray

Relbunium microphyllum

(A. Gray) Hemsl.

Arb/C,M-C/720

Suf/E,C,M-R/261

$\mathrm{Hp} / \mathrm{E}, \mathrm{R} / 944$

Hp/R,C,M-R/517

\section{RUTACEAE}

Ptelea trifoliata L.

Arb/R/1171

SAPINDACEAE

Dodonaea viscosa (L.) Jacq.

Arb/C/730

\section{SAXIFRAGACEAE}

Heuchera mexicana Schaffn.

\section{SCROPHULARIACEAE}

Aureolaria greggii (Wats.) Perman

Bacopa procumbens (Mill.) Greenm.

Castilleja integra A. Gray

Castilleja lanata A. Gray

Castilleja lithospermoides H.B.K.

Castilleja mexicana (Hemsl.) A. Gray

Castilleja tenuiflora Benth.

Lamourouxia dasyantha

(Cham. \& Schlecht.) Ernst

Leucophyllum frutescens

(Berl.) I. M. Johnst.

Penstemon barbatus (Cav.) Roth

Penstemon lanceolatus Benth.

Penstemon potosinus Lundell

Penstemon roseus (Sweet) G. Don

Seymeria scabra A. Gray

$\mathrm{Hp} / \mathrm{E} / 936$

$\mathrm{Hp} / \mathrm{R} / 403$

$H p / P, E, R, C$,

$P-E / 955$

$\mathrm{Hp} / \mathrm{C} / 401$

$\mathrm{Hp} / \mathrm{C} / 1014$

$\mathrm{Hp} / \mathrm{E}, \mathrm{R} / 1038$

$\mathrm{Hp} / \mathrm{E} / 357$

$\mathrm{Hp} / \mathrm{E}, \mathrm{R} / 824$

Suf/E,C,R-/1142

Arb/R/400

$\mathrm{Hp} / \mathrm{E}, \mathrm{R} / 807$

$\mathrm{Hp} / \mathrm{R}, \mathrm{R}-\mathrm{C} / 904$

Hp/M-R/521

$\mathrm{Hp} / \mathrm{E} / 937$

$\mathrm{Hp} / \mathrm{E} / 336$

SOLANACEAE

Bouchetia erecta DC.

Datura stramonium L.

Jaltomata procumbens

(Cav.) J. L. Gentry

Lycium berlandieri Dunal

Nicotiana glauca Graham.

Nicotiana trigonophylla Dunal

Physalis caudella Standl.

Physalis virginiana Mill.

Solanum nigrescens Mart. \& Gal.

Solanum rostratum Dunal

Solanum stoloniferum Schlecht.

Solanum verrucosum Schlecht.
Hp/P,C,P-E/968

$\mathrm{Ha} / \mathrm{M}, \mathrm{M}-\mathrm{R} / 981$

$\mathrm{Ha} / \mathrm{M}-\mathrm{C} / 717$

Arb/M-R/914

$\mathrm{Ar} / \mathrm{C} / 743$

$\mathrm{Hp} / \mathrm{M}, \mathrm{M}-\mathrm{R} / 885$

$\mathrm{Hp} / \mathrm{E} / 344$

$\mathrm{Ha} / \mathrm{C} / 570$

$\mathrm{Ha} / \mathrm{E}, \mathrm{R}, \mathrm{C} / 571$

$\mathrm{Ha} / \mathrm{M} / 311$

$\mathrm{Hp} / \mathrm{E} / 244$

$\mathrm{Hp} / \mathrm{E} / 1155$ 


\section{FAMILIAS}

Especies

Clave*

\section{URTICACEAE}

Parietaria pensylvanica Muhl.

$\mathrm{Ha} / \mathrm{C} / 963$

\section{VERBENACEAE}

Verbena ambrosifolia Rydb.

Verbena canescens H.B.K.

Verbena gracilis Desf.

Verbena menthaefolia Benth.

\section{ZYGOPHYLLACEAE}

Larrea tridentata (DC.) Coville

\section{LILIOPSIDA}

\section{AGAVACEAE}

Agave filifera Salm

Agave lechuguilla Torr.

Agave salmiana ssp. crassispina

(Trel.) Gentry

Agave striata Zucc.

Dasylirion cedrosanum Trel.

Nolina texana Wats.

Yucca carnerosana

(Trel.) McKelvey

Yucca decipiens Trel.

Yucca filifera Chabaud

\section{BROMELIACEAE}

Tillandsia erubescens Schlecht.

Tillandsia recurvata (L.) L.

Tillandsia usneoides (L.) L.

\section{COMMELINACEAE}

Aneilema geniculata Jacq.

Commelina dianthifolia DC.

Tradescantia crassifolia Cav.

\section{CYPERACEAE}

Eleocharis montana

(H.B.K.) Roem. \& Schult

Bulbostylis juncoides (Vahl) Kük.

Carex schiedeana Kunze

Cyperus esculentus L.

Cyperus seslerioides H.B.K.

Cyperus spectabilis Link
$H p / R-E / 369$

$\mathrm{Hp} / \mathrm{R}, \mathrm{C}-\mathrm{R} / 1098$

$\mathrm{Hp} / \mathrm{M}-\mathrm{R} / 542$

$\mathrm{Hp} / \mathrm{C}, \mathrm{P}-\mathrm{E} / 1076$

Arb/M,R/1024

Ros/E,P,E-P/345

Ros/R/1069

$\operatorname{Ros} / \mathrm{C}, \mathrm{M} / 1181$

Ros/R/895

Ros/R/1067

Ros/R,E,P-E/1102

$\mathrm{Ar} / \mathrm{R} / 1068$

$\mathrm{Ar} / \mathrm{M}, \mathrm{C}, \mathrm{M}-\mathrm{C} / 1052$

$\mathrm{Ar} / \mathrm{M}, \mathrm{C}, \mathrm{M}-\mathrm{C} / 1054$

$\mathrm{Ep} / \mathrm{E} / 1031$

$\mathrm{Ep} / \mathrm{E} / 884$

$\mathrm{Ep} / \mathrm{E} / 1030$

$\mathrm{Hp} / \mathrm{R}, \mathrm{E}-\mathrm{R} / 343$

$\mathrm{Hp} / \mathrm{E}, \mathrm{E}-\mathrm{P} / 346$

$\mathrm{Hp} / \mathrm{E} / 249$

\section{$\mathrm{Hp} / \mathrm{C} / 635$}

$\mathrm{Hp} / \mathrm{P} / 578$

$\mathrm{Hp} / \mathrm{R}, \mathrm{E}, \mathrm{R}-\mathrm{E} / 463$

$\mathrm{Hp} / \mathrm{E} / 390$

$\mathrm{Hp} / \mathrm{P}, \mathrm{P}-\mathrm{E} / 649$

$\mathrm{Hp} / \mathrm{P}, \mathrm{C} / 695$

\section{IRIDACEAE}

Sisyrinchium scabrum Schlecht. \& Cham. Hp/R/331

Sisyrinchium tenuifolium.

Humb. \& Bonpl. ex Willd.

$\mathrm{Hp} / \mathrm{E}, \mathrm{C} / 442$

\section{JUNCACEAE}

Juncus tenuis Willd.

$\mathrm{Hp} / \mathrm{P} / 582$

\section{LILIACEAE}

Aloe barbadensis Mill.
FAMILIAS
Especies
Clave*

Allium glandulosum Link \& Otto

Asphodelus fistulosus L.

Echeandia flavescens

(Schult. \& Schult. f.) Cruden

Nothoscordum bivalve (L.) Britton

$\mathrm{Hp} / \mathrm{P}, \mathrm{R}-\mathrm{E} / 601$

$\mathrm{Hp} / \mathrm{R}, \mathrm{E} / 993$

$\mathrm{Hp} / \mathrm{C}, \mathrm{R} / 442$

$\mathrm{Hp} / \mathrm{R}, \mathrm{M} / 292$

\section{ORCHIDACEAE}

Spiranthes cinnabarina

(Llave \& Lex.) Hemsl.

$\mathrm{Hp} / \mathrm{R}, \mathrm{C} / 1122$

\section{POACEAE(GRAMINEAE)}

Agrostis semiverticillata (Forsk.) C. Chr. Hp/C,P-E/664

Aristida curvifolia Fourn.

$\mathrm{Ha} / \mathrm{R} / 930$

Aristida divaricata Humb. \& Bonpl.

Aristida glauca (Nees) Walp.

Aristida orcuttiana Vasey

Bothriochloa wrightii (Hack.) Henr.

Bouteloua barbata Lag.

Bouteloua curtipendula (Michx.) Torr.

$\mathrm{Hp} / \mathrm{C}, \mathrm{M}$,

$\mathrm{P}-\mathrm{E}-\mathrm{R} / 593$

$\mathrm{Hp} / \mathrm{R}, \mathrm{E} / 860$

$\mathrm{Hp} / \mathrm{R} / 600$

$\mathrm{Hp} / \mathrm{R}, \mathrm{R}-\mathrm{M} / 97$

$\mathrm{Ha} / \mathrm{R}, \mathrm{M}, \mathrm{C} / 512$

$\mathrm{Hp} / \mathrm{R}, \mathrm{M}-\mathrm{R}$,

$\mathrm{E}-\mathrm{P} / 465 \mathrm{a}$

Bouteloua gracilis (H.B.K.) Lag.

Brachiaria meziana Hitchc.

Brachypodium mexicanum

(Roem. \& Schult.) Link

Bromus anomalus Rupr. ex Fourn.

Bromus carinatus Hook. \& Arn.

Buchloe dactyloides (Nutt.) Engelm.

Chloris submutica H.B.K.

Chloris virgata $\mathrm{Sw}$.

Dasyochloa pulchella

(H.B.K.) Willd. ex Rydb.

Elymus longifolius (J.G. Sm.) Gould

Enneapogon desvauxii Beauv.

Eragrostis intermedia Hitchc.

Eragrostis mexicana (Hornem.) Link

Eragrostis pectinacea (Michx.) Nees

Erioneuron avenaceum

(H.B.K.) Tateoka

Hilaria cenchroides H.B.K.

Leptochloa dubia (H.B.K.) Nees

Lycurus phalaroides H.B.K.

Lycurus phleoides H.B.K.

$\mathrm{Hp} / \mathrm{R}, \mathrm{C}, \mathrm{P} / 586$

$\mathrm{Hp} / \mathrm{C} / 726$

$\mathrm{Hp} / \mathrm{R}, \mathrm{P}-\mathrm{E}-\mathrm{R} / 868$

$\mathrm{Hp} / \mathrm{C}, \mathrm{R}, \mathrm{M}-\mathrm{R} / 898$

$\mathrm{Hp} / \mathrm{R} / 831$

$\mathrm{Hp} / \mathrm{M} / 504$

$\mathrm{Hp} / \mathrm{C}, \mathrm{P}-\mathrm{E}-\mathrm{R} / 855$

$\mathrm{Ha} / \mathrm{C} / 728$

$\mathrm{Hp} / \mathrm{M}-\mathrm{R} / 972$

$\mathrm{Hp} / \mathrm{R}-\mathrm{M}$,

$\mathrm{P}-\mathrm{E}-\mathrm{R} / 896$

$\mathrm{Ha} / \mathrm{M} / 509$

$\mathrm{Hp} / \mathrm{P}, \mathrm{C}, \mathrm{P}-\mathrm{E}-\mathrm{R} / 857$

$\mathrm{Hp} / \mathrm{C}, \mathrm{M} / 687$

$\mathrm{Ha} / \mathrm{C}, \mathrm{P} / 636$

$\mathrm{Ha} / \mathrm{C} / 509$

$\mathrm{Hp} / \mathrm{C}, \mathrm{R}, \mathrm{M} / 506$

$\mathrm{Hp} / \mathrm{C}, \mathrm{M}-\mathrm{R} / 647$

$\mathrm{Hp} / \mathrm{C}, \mathrm{M}, \mathrm{P}, \mathrm{E} / 754$

$\mathrm{Hp} / \mathrm{R}, \mathrm{C}, \mathrm{E}, \mathrm{M}-\mathrm{R}$,

$\mathrm{P}-\mathrm{E}-\mathrm{R} / 391$

Metcalfia mexicana (Scribn.) Conert Muhlenbergia distans Swallen

Muhlenbergia dubia Fourn.

Muhlenbergia glauca (Nees) Mez

$\mathrm{Hp} / \mathrm{R} / 466 \mathrm{a}$

Hp/R,P,P-E-R,

$P-E / 467$

$\mathrm{Hp} / \mathrm{R} / 479$

$\mathrm{Hp} / \mathrm{P} / 868$

Muhlenbergia microsperma (DC.) Kunth $\mathrm{Ha} / \mathrm{C} / 692$

Muhlenbergia pubescens

(H.B.K.) Hitchc.

Muhlenbergia rigida (H.B.K.) Kunth
$\mathrm{Hp} / \mathrm{P} / 597$

$\mathrm{Hp} / \mathrm{R}, \mathrm{C}, \mathrm{P}$, 

FAMILIAS
Especies
Clave*

Panicum obtusum H.B.K.

Piptochaetium brevicalyx

(Fourn.) Ricker

Piptochaetium fimbriatum

(H.B.K.) Hitchc.

Scleropogon brevifolius Phil.

\section{FAMILIAS \\ Especies}

Clave*
$\mathrm{Hp} / \mathrm{M}-\mathrm{R} / 818$

$\mathrm{Hp} / \mathrm{M} / 678$

$\mathrm{Hp} / \mathrm{C}-\mathrm{R} / 567$

Setaria macrostachya H.B.K. Sporobolus airoides (Torr.) Torr.

Stipa eminens Cav.

Stipa neomexicana (Thurb.) Scribn.

Stipa robusta (Vasey) Scribn.

$\mathrm{Hp} / \mathrm{P}, \mathrm{E}, \mathrm{P}-\mathrm{E} / 575 \mathrm{~b}$

$\mathrm{Hp} / \mathrm{C}-\mathrm{R} / 567$
$\mathrm{Hp} / \mathrm{M}, \mathrm{C} / 690$

$\mathrm{Hp} / \mathrm{M}-\mathrm{R} / 547$

$\mathrm{Hp} / \mathrm{C} / 693$

$\mathrm{Hp} / \mathrm{C} / 926$

$\mathrm{Hp} / \mathrm{C} / 693$

Hp/R,M-R,

P-E-R/851

Claves: Formas vitales: Ar, arbóreo; Arb, arbusto; Bi, bienal; Cra, crasicaule; Ep, epífita; Epp, epiparásita; Esc, escandente; $\mathrm{Ha}$, herbácea anual; $\mathrm{Hp}$, herbácea perenne; Ras, rastrera; Ros, rosetófilo; Sar, subarbórea; Suf, sufrútice; Tre, trepadora. Tipos de vegetación: $C$, matorral crasicaule; $E$, encinar arbustivo; $M$, matorral desértico micrófilo; $P$, piñonar; $R$, matorral desértico rosetófilo. Números de colecta: Número de colecta que el primer autor registró en su libreta de campo durante la etapa de colecta, por razones de espacio sólo se incluye un número, pero en promedio se tienen dos números por cada especie. Para todos los datos del sitio son: México, estado de San Luis Potosí, municipio de Charcas, Sierra Monte Grande, 2,200 a 2,800 m s.n.m., entre el mes de agosto de 1989 y el de septiembe de 1990. * Fuera de cultivo.

\section{Literatura citada}

Aguirre R.J.R. 1970. Estudio sobre el cardenche (Opuntia imbricata (Haw.) DC.), en la región ganadera del noroeste de San Luis Potosí. Tesis profesional. Escuela Nacional de Agricultura. Chapingo, México. 62 pp.

Aldrete E. y Aguirre J.R. 1981. El matorral rosetófilo del noreste del estado de Zacatecas $\left(24^{\circ} 00^{\prime}-24^{\circ} 52^{\prime}\right.$ latitud norte y $100^{\circ} 49^{\prime}-102^{\circ} 00^{\prime}$ longitud oeste). Memorias del VII Congreso Mexicano de Botánica. Morelia, Michoacán. pp: 336-337.

Anónimo. 1971. Carta topográfica F-14-A33. Esc. 1:50,000. Comisión de Estudios del Territorio Nacional, Secretaría de Programación y Presupuesto. México, D.F.

Anónimo. 1975. Carta topográfica F-14-A33. Esc. 1:250,000. Comisión de Estudios del Territorio Nacional, Secretaría de Programación y Presupuesto. México, D.F.

Anónimo. s.f. Datos climáticos mensuales de la estación meteorológica del ejido de Coyotillos, Mpio. de Charcas, S.L.P. Subdelegación de Infraestructura Hidráulica, Secretaría de Agricultura y Recursos Hidraúlicos. San Luis Potosí, S.L.P.

Balleza J.J. 1993. Gramíneas de Zacatecas, México. Lista de especies y clave para géneros. Tesis de maestría en ciencias. Colegio de Postgraduados. Montecillo, México 108 pp.

Becerra N. 1971. Comportamiento de otinos en pastoreo en vegetación árida del noroeste de San Luis Potosi. Tesis profesional. Escuela Nacional de Agricultura. Chapingo, México. 60 pp.

Bravo H. 1978. Las cactáceas de México I. Universidad Nacional Autónoma de México. México, D.F. 404 pp.

Brummitt R.K. and Powell C.E. 1992. Authors of plants names. Royal Botanic Gardens. Kew, England. 732 pp.
Calderón G. 1960. Notas sobre la flora y la vegetación del estado de San Luis Potosí. VII. Vegetación del valle de San Luis Potosí. Acta Científica Potosina 4(5-12)

Cronquist A. 1981. An integrated system of classification of flowering plants. Columbia University Press. Nueva York. 262 pp.

Del Castillo S. 1982. Estudio ecológico de Ferocactus histrix (DC.) Lindsay. Tesis profesional. Escuela Nacional de Estudios Profesionales Iztacala. Universidad Nacional Autónoma de México. Tlalnepantla, México. 228 pp.

Flores F.J.L. 1985. Evaluación de tierras en el área de estudios del CREZAS-CP. Tesis profesional. Universidad Autónoma Chapingo. Chapingo, México. 40 pp.

González M. 1975. Distribución espacial y su interpretación sucesional en el noreste del estado de Zacatecas. Tesis profesional. Escuela Nacional de Agricultura. Chapingo, México. 263 pp.

Hernández R.E.M. 1985. Cactáceas de San Luis Potosí, S.L.P. México. Instituto de Investigaciones de Zonas Desérticas. Universidad Autónoma de San Luis Potosí. San Luis Potosí, S.L.P. 91 pp.

Juárez J.M.A., Reyes J.A. y Andrade A.J.A. Flora útil de tres tipos de vegetación del altiplano potosino-zacatecano. Geografía Agrícola. (en prensa).

Madrigal S.X. 1986. Notas para el curso de botánica IV. Angiospermas. Edición mimeografiada. Universidad Michoacana de San Nicolás de Hidalgo. Morelia, Mich. México. 166 pp.

Marroquín J.S., Borja G. Velázquez R. y de la Cruz J.A. 1981. Estudio ecológico dasonómico de las zonas áridas del norte. 2a edición. Instituto Nacional de Investigaciones Forestales. México, D.F. 166 pp. 
Molina M.C. 1990. Caracterización del banco de semillas de un pastizal con diferente grado de deterioro. Tesis de maestría en ciencias. Colegio de Postgraduados. Chapingo, México. 87 pp.

Passini M.F. 1982. Les forêts de Pinus cembroides au Mexique. Mission Archéologique et Ethnologique Française au Mexique. París. 373 pp.

Pinkava D.J. 1984.Vegetation and flora of Bolson de Cuatro Ciénegas Region, Coah., Mexico. IV. Summary, endemisms and corrected catalogue. Journal of Arizona-Nevada Academy of Science 19(1):23-47.

Ramamoorthy T.P. y Elliot M. 1993. Mexican Lamiacea: diversity, distribution, endemism, and evolution. En: Ramamoorthy T.P., Bye R., Lot A.J. y Eds. Biological diversity of Mexico: Origins and distribution. Oxford University Press. New York, USA. pp. 513-539.

Robledo M.A. 1990. Aspectos ecológicos y etnobotánicos del orégano silvestre en el altiplano potosino-zacatecano. Tesis profesional. Escuela Nacional de Estudios Profesionales Iztacala; Universidad Nacional Autónoma de México. Tlalnepantla, México. $116 \mathrm{pp}$.

Rzedowski J. 1955. Notas sobre la flora y la vegetación de San Luis Potosí. II. Estudio de diferencias florísticas y ecológicas condicionadas por ciertos tipos de substrato geológico. Ciencia 15:141-147

Rzedowski J. 1956. Notas sobre la flora y la vegetación de San Luis Potosí. III. Vegetación de la región de Guadalcázar. Anales del Instituto de Biología. Universidad Nacional Autónoma de México 27:169-228.

Rzedowski J. 1957.Vegetación de las partes áridas de los estados de San Luis Potosí y Zacatecas. Revista de la Sociedad Mexicana de Historia Natural 8:49-101.
Rzedowski J. 1959. Las colecciones botánicas de Wilhelm (José Guillermo) Schaffner en San Luis Potosí. I. Acta Cientifica Potosina 3:99-121.

Rzedowski J. 1960. La vegetación como indicador de rocas ígneas en la parte árida del estado de San Luis Potosí. Boletín de la Sociedad Geológica Mexicana. 23:79-84.

Rzedowski J. 1965.Vegetación del estado de San Luis Potosí. Acta Científica Potosina 5:5-291.

Rzedowski J. 1972. Contribuciones a la fitogeografía florística e histórica de México. III. Algunas tendencias en la distribución geográfica y ecológica de las Compositae mexicanas. Ciencia 27:123-132.

Rzedowski J. 1978. Vegetación de México. Limusa. México, D.F. 432 pp.

Rzedowski J. 1991. Diversidad y orígenes de la flora fanerogámica de México. Acta Botánica Méxicana 14:3-21.

Rzedowski J. y Calderón G. 1957.Notas sobre la flora y la vegetación del estado de San Luis Potosí. VI. Algunas plantas nuevas para la flora del estado. Acta Científica Potosina 1:199-218.

Salas S. 1987. La familia Compositae en la zona árida del estado de San Luis Potosí, México. Instituto de Investigaciones de Zonas Desérticas. Universidad Autónoma de San Luis Potosí. San Luis Potosí, S.L.P. 2 vols. 273 pp.

Sánchez F.R. 1989. Leguminosas del municipio de San Luis Potosí, México. Instituto de Investigaciones de Zonas Desérticas. Universidad Autónoma de San Luis Potosí. San Luis Potosí, S.L.P. 75 pp.

Tryon M. y Tryon A.F. 1982. Ferns and allied plants. SpringVerlag. Nueva York. 857 pp.

Villarreal Q.J.A. 1994. Flora vascular de la Sierra de la Paila, Coahuila, México. Sida 16:109-138. 